\title{
El envío de mensajes de texto móvil podría mejorar la adherencia a la medicación en pacientes con enfermedades crónicas
}

Mobile telephone text messaging could improve medication adherence in chronic disease

\section{Objetivos}

Evaluar el efecto del envío de mensajes de texto por telefonía móvil sobre la adherencia a la medicación en adultos con enfermedades crónicas.

\section{Diseño y fuente de datos}

Meta-análisis. La búsqueda incluyó las bases de datos MEDLINE, EMBASE, Cochrane Central Register of Controlled Trials, PsycINFO, y CINHL (desde su inicio y hasta Enero de 2015) y listas de referencias de los artículos. Los datos fueron analizados en marzo de 2015.

\section{Selección de estudios}

Ensayos clínicos aleatorizados (ECA) que evaluaron el envío de mensajes de texto como intervención para promover la adherencia a la medicación en adultos con enfermedades crónicas.
Thakkar J, y col. JAMA Intern Med 2016;176:340-9.

\section{Extracción de datos}

De manera independiente dos autores extrajeron información acerca de las características de los estudios, mensajes de texto y la medición de resultados, según protocolo.

\section{Resultados Principales}

La mediana de duración de la intervención fue de 12 semanas, siendo el auto-reporte el método más usado para evaluar la a-dherencia. Los resultados principales se resumen en la tabla 1. El efecto beneficioso no sufrió influencias significativas a las características del estudio (duración de la intervención o tipo de enfermedad) o a las características del mensaje de texto (personalización, comunicación de dos vías, o frecuencia diaria de mensajes). El envío de mensajes de texto, duplicó aproximadamente la chance de presentar adherencia a la medicación. Este incremento se tradujo en un aumento de la tasa de adherencia desde $50 \%$ (asumiendo éste porcentaje como línea de base en pacientes con enfermedad crónica) a $67,8 \%$; o un aumento absoluto del $17,8 \%$.

Tabla 1. Efecto de los mensajes de texto móvil sobre la adherencia a la toma de medicación.

\begin{tabular}{|l|c|c|c}
\hline $\begin{array}{c}\text { Número de estudios } \\
\text { (nro. de pacientes } \\
\text { incluidos) }\end{array}$ & Exposición/Comparación & $\begin{array}{c}\text { Resultado } \\
\text { OR (IC 95\%) }\end{array}$ & $\begin{array}{c}\text { Análisis de Sensibilidad } \\
\text { OR (IC 95\%) }\end{array}$ \\
$\begin{array}{l}16 \text { Ensayos clínicos aleatorizados } \\
(2.742)\end{array}$ & $\begin{array}{c}\text { Envío de mensajes de texto/ } \\
\text { Toma habitual de medicación. }\end{array}$ & $\begin{array}{c}2,11(1,52 \mathrm{a} 2,93) ; \\
\mathrm{p}<0.001 ; 12: 62 \%\end{array}$ & $\begin{array}{c}\text { Ajuste para sesgo de publicación } \\
\text { (IC 95\%) }\end{array}$ \\
\hline
\end{tabular}

OR: Odds Ratio; IC: Intervalo de Confianza; P: p-valor del estadístico calculado; I2: Heterogeneidad.

\section{Conclusiones}

Si bien se duplicó la chance de mejora en la adherencia a la medicación, estos resultados deberían ser interpretados con cuidado, dada la corta duración de los ensayos clínicos, y la confianza en el auto-reporte como medida de la adherencia a la medicación. Estudios futuros serían necesarios para determinar las características del envío de mensajes de texto como intervención, así como poblaciones apropiadas de pacientes, efectos sostenidos e influencias en resultados clínicos.

Fuente de financiamiento/conflicto de interés de los autores: Los autores reportaron becas de posgrado, fellowship como investigador principal del $\mathrm{Na}$ tional Health and Medical Research Council y financiación de Roche, Amgen, y Novartis, el grant APP1061793 del National Heart Foundation (NHF) y el grant 1033478 del "NHF and Sydney Medical Foundation Chapman Fellowship". No se reportaron otros conflictos de interés.

\section{Comentario}

La Organización Mundial de la Salud (OMS) define adherencia "como el grado en que el comportamiento de una persona, por ejemplo al tomar un medicamento, seguir un régimen alimentario y ejecutar cambios del modo de vida, se corresponde con las recomendaciones acordadas con un prestador de asistencia sanitaria". Este resulta el segundo problema más grande sin resolver en el cuidado de la salud, y es por eso que se han hecho múltiples intervenciones para mejorarla, siendo la mensajería móvil una de ellas. Respecto de las limitaciones de este metaanálisis podemos señalar: a) una gran heterogeneidad; b) que los estudios incluidos no tuvieron población mayor a 65 años, teniendo este grupo etario una alta prevalencia de enfermedades crónicas; c) que el tiempo de evaluación medio fue de 12 semanas, lo cual resulta insuficiente para poder evaluar adherencia en enfermedades crónicas, y; d) el uso de auto-reporte para determinar adherencia, ya que existe evidencia indicando que este mecanismo podría sobreestimarla hasta en un $200 \%{ }^{1}$. Es así que no sería adecuado tomar decisiones intervencionistas basadas en asunciones poco precisas. Actualmente se conoce que la adherencia está influenciada simultáneamente por muchos factores, y que la habilidad de los pacientes para seguir el tratamiento indicado es afectada por más de una barrera, como ser factores socioeconómicos, individuales (motivación, olvidos, creencias del usuario), costos de la medicación, el equipo/sistema de salud, características de la enfermedad y complejidad y tipo de tratamiento ${ }^{2}$. Para mejorarla, se debe incidir sobre todos estos factores en conjunto, y es poco probable que una sola intervención aislada como es el envío de mensajes de texto, pueda derivar en un beneficio clínico.

\section{Conclusiones del comentador}

Si bien el envío de mensajes de texto podría mejorar la adherencia a la medicación en enfermedades crónicas, el principal esfuerzo debe ser el de identificar barreras potenciales en el tratamiento, valorar la motivación del paciente para tomar la medicación prescripta, aumentar su conocimiento acerca de los beneficios potenciales y la confianza en los proveedores del sistema de salud, así como seguir buscando soluciones multifactoriales integradoras que puedan derivar en un aumento efectivo de la adherencia a la medicación, y en consecuencia, resultados clínicamente relevantes.

Sonia E. Benítez y Rodrigo R. Cano García [ Servicio de Informática Médica del Hospital Italiano de Buenos Aires. sonia.benitez@ hospitalitaliano.org. ar, rodrigo.cano.med@gmail.com]

Benítez SE. Cano García RR. El envío de mensajes de texto móvil podría mejorar la adherencia a la medicación en pacientes con enfermedades crónicas. Evid Act Pract Ambul. 2017;20(1):9. Comentado de: Thakkar J, y col. Mobile telephone text messaging for medication adherence in chronic disease: a meta-analysis. JAMA Intern Med. 2016;176(3):340-349. PMID:26831740. 